Modern Physics Letters A

(C) World Scientific Publishing Company

\title{
INELASTIC MESON-MESON SCATTERING IN HADRONIC MATTER
}

\author{
XIAO-MING XU \\ Department of Physics, Shanghai University, 99 Shangda Road, \\ Baoshan, Shanghai 200444, China \\ xmxu@mail.shu.edu.cn \\ H. J. WEBER \\ Department of Physics, University of Virginia, Mccormick Road, \\ Charlottesville, VA 22904, USA \\ Received (Day Month Year) \\ Revised (Day Month Year)
}

\begin{abstract}
We review studies of inelastic meson-meson scattering. In nonperturbative schemes with chiral-perturbation-theory Lagrangians and in models based on effective meson Lagrangians, inelastic meson-meson scattering leads to the successful identification of resonances in meson-meson reactions, adequate inclusion of final state interactions in particle decays, and so on. For mesons of which each consists of a quark and an antiquark, inelastic meson-meson scattering may be caused by quark-antiquark annihilation, quarkantiquark creation, quark interchange, and quark-antiquark annihilation and creation. In transition amplitudes for meson-meson scattering mesonic quark-antiquark relativemotion wave functions depend on hadronic matter, and transition potentials are given in perturbative quantum chromodynamics. Via transition amplitudes the cross sections for inelastic meson-meson scattering depend on the temperature of hadronic matter. Some prominent temperature dependence of the cross sections has been found. Inelastic meson-meson scattering becomes even more significant in proton-proton collisions and lead-lead collisions at the Large Hadron Collider.
\end{abstract}

Keywords: inelastic meson-meson scattering; transition amplitude; $T$-matrix element; hadronic matter.

PACS Nos.: 13.75.Lb; 13.85.Hd; 12.39.Pn; 12.39.Fe

\section{Introduction}

Meson-meson scattering is an important tool in studying resonances. Resonances are produced by the strong interaction of the colliding mesons. This amounts to two-to-one meson-meson scattering, for example, $\pi \pi \rightarrow \rho$ and $\pi K \rightarrow K^{*}$.

When the center-of-mass energy of two pions increases from the sum of two pion masses, elastic pion-pion scattering takes place. When the energy exceeds the sum of two kaon masses (four pion masses), a $K \bar{K}$ pair (two more pions) may be produced. The scattering process becomes elastic $\pi \pi$ scattering, elastic $K \bar{K}$ scat- 
tering, or elastic four-pion scattering. The connection between elastic $\pi \pi$ scattering and elastic $K \bar{K}$ scattering is the inelastic scattering $\pi \pi \leftrightarrow K \bar{K}$. A couped-channel formalism is the appropriate framework for describing this meson-meson scattering. Inelastic two-to-two meson-meson scattering is important in studying meson-meson scattering, ${ }^{\sqrt[1]{3}}$ photon-photon reactions, ${ }^{\sqrt[3]{6}} \mathrm{~J} / \psi$ decays, ${ }^{[7} \sqrt{9}$ etc.

Recently, the LHCb Collaboration at the Large Hadron Collider (LHC) has measured $p p$ collisions to examine the symmetry under the combined charge-conjugation and parity-transformation operations ${ }^{10} 13(\mathrm{CP})$ in the decays $B^{ \pm} \rightarrow \pi^{ \pm} \pi^{+} \pi^{-}$, $B^{ \pm} \rightarrow \pi^{ \pm} K^{+} K^{-}, B^{ \pm} \rightarrow K^{ \pm} \pi^{+} \pi^{-}$, and $B^{ \pm} \rightarrow K^{ \pm} K^{+} K^{-}$. The CP asymmetry observed in the decay modes is unexpectedly large in some kinematical regions of the final mesons. The large $\mathrm{CP}$ violation has been attributed to weak interactions, resonances of final mesons, and $\pi \pi \leftrightarrow K \bar{K}$ !14

Quark-gluon plasmas are created in Au-Au collisions at the Relativistic Heavy Ion Collider and in $\mathrm{Pb}-\mathrm{Pb}$ collisions at the LHC. The plasmas eventually transform into hadronic matter at the critical temperature. Hadronic matter changes until kinetic freeze-out occurs. The change of hadronic matter is governed by hadron-hadron scattering. All possible hadron-hadron scattering occurs in hadronic matter. Many experiments have been done for nucleon-nucleon collisions, $p \bar{p}$ collisions, and mesonnucleon collisions. ${ }^{[5]}$ However, the role these collisions play in hadronic matter is still largely unknown. Experimental data on inelastic meson-meson scattering are scarce. Therefore, many hadron-hadron collisions in hadronic matter and even in vacuum have to be studied theoretically. This is a huge task for theorists!

Mesons are the dominant species in hadronic matter. The usual scattering is twoto-one meson-meson scattering and two-to-two meson-meson scattering. In leadlead collisions and in xenon-xenon collisions at the LHC, the meson momentum measured by the ATLAS Collaboration, the CMS Collaboration, and the ALICE Collaboration goes up to $1000 \mathrm{GeV} / c \frac{17] 19}{10}$ meson of such large momenta in collision with another meson in hadronic matter may yield three or more mesons. Two-to-three meson-meson scattering affects the chemical equilibrium of hadronic matter, and causes energy loss of the large-momentum meson. Therefore, we need to study two-to-three meson-meson scattering in hadronic matter as well.

We review inelastic meson-meson scattering in vacuum and in hadronic matter. Inelastic meson-meson scattering in the coupled-channel unitary approaches and the inverse amplitude method are discussed in Sec. 2. Cross sections from effective meson Lagrangians are introduced in Sec. 3. We talk about meson-meson reactions in Secs. 4-9 for the following processes: quark-antiquark annihilation, quark-antiquark creation, quark-antiquark annihilation and creation, and quark interchange. A summary is given in the last section.

\section{Inelastic Meson-Meson Scattering in Coupled Channels}

Describing mesons as quark-antiquark currents, the most general effective Lagrangian that respects Lorentz invarinace, parity symmetry, and chiral symmetry 
was put forward by Gasser and Leutwyler in 1984.20 This is the Lagrangian of $S U(2)$ chiral perturbation theory $(\chi \mathrm{PT})$. Including the strange quark, $\mathrm{SU}(3) \chi \mathrm{PT}$ was established in Ref. ${ }^{21}$ Furthermore, including the $\eta^{\prime}$ meson, $U(3) \chi \mathrm{PT}$ was established in Refs. ${ }^{22}$ The $\chi \mathrm{PT}$ Lagrangians are given in powers of external momenta $p$ and quark masses. The Lagrangian to next-to-leading order in $S U(3) \chi \mathrm{PT}$ is

$$
\mathcal{L}=\mathcal{L}_{2}+\mathcal{L}_{4} .
$$

The term $\mathcal{L}_{2}$ is of order $p^{2}$, and is given by

$$
\mathcal{L}_{2}=\frac{f_{0}^{2}}{4}<\partial_{\mu} U^{+} \partial^{\mu} U+M\left(U+U^{+}\right)>
$$

where $f_{0}$ is the pion decay constant, $M$ is the quark-mass-dependent matrix,$\frac{1}{1}$ and the angular brackets stand for the trace of the quantity enclosed. The matrix $U=$ $\exp \left(i \sqrt{2} \Phi / f_{0}\right)$ displays the pseudo Goldstone boson fields by

$$
\Phi(x)=\left(\begin{array}{ccc}
\frac{1}{\sqrt{2}} \pi^{0}+\frac{1}{\sqrt{6}} \eta & \pi^{+} & K^{+} \\
\pi^{-} & -\frac{1}{\sqrt{2}} \pi^{0}+\frac{1}{\sqrt{6}} \eta & K^{0} \\
K^{-} & \bar{K}^{0} & -\frac{2}{\sqrt{6}} \eta
\end{array}\right),
$$

where $x$ are the space-time coordinates. The term $\mathcal{L}_{4}$ is of order $p^{4}$ :

$$
\begin{aligned}
\mathcal{L}_{4} & =L_{1}<\partial_{\mu} U^{+} \partial^{\mu} U>^{2}+L_{2}<\partial_{\mu} U^{+} \partial_{\nu} U><\partial^{\mu} U^{+} \partial^{\nu} U> \\
& +L_{3}<\partial_{\mu} U^{+} \partial^{\mu} U \partial_{\nu} U^{+} \partial^{\nu} U>+L_{4}<\partial_{\mu} U^{+} \partial^{\mu} U><U^{+} M+M^{+} U> \\
& +L_{5}<\partial_{\mu} U^{+} \partial^{\mu} U\left(U^{+} M+M^{+} U\right)>+L_{6}<U^{+} M+M^{+} U>^{2} \\
& +L_{7}<U^{+} M-M^{+} U>^{2}+L_{8}<M^{+} U M^{+} U+U^{+} M U^{+} M>,
\end{aligned}
$$

where $L_{h}(h=1, \cdots, 8)$ are constants.

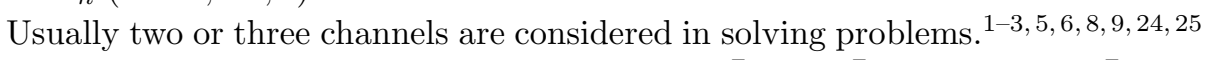
For example, the two-channel case is $\pi \pi \rightarrow \pi \pi, K \bar{K} \rightarrow K \bar{K}$, and $\pi \pi \leftrightarrow K \bar{K}$, and the three-channel case is $\pi \eta \rightarrow \pi \eta, K \bar{K} \rightarrow K \bar{K}, \pi \eta^{\prime} \rightarrow \pi \eta^{\prime}, \pi \eta \leftrightarrow K \bar{K}, \pi \eta \leftrightarrow \pi \eta^{\prime}$, and $K \bar{K} \leftrightarrow \pi \eta^{\prime}$. Denote by $N$ the number of channels involved. Elastic mesonmeson scattering is indicated by $A_{i}+B_{i} \rightarrow A_{i}+B_{i}$ with $i=1, \cdots, N$. In the coupled-channel unitary approaches one needs to calculate potentials from $\mathcal{L}_{2}$ :

$$
V_{j i}=<A_{j} B_{j}\left|\mathcal{L}_{2}\right| A_{i} B_{i}>
$$

From the Lippmann-Schwinger equations the $T$-matrix elements for $A_{i}+B_{i} \rightarrow$ $A_{j}+B_{j}$ with $j=1, \cdots, N$ satisfy

$$
T_{j i}=V_{j i}+\sum_{k=1}^{N} V_{j k} G_{k k} T_{k i},
$$

where $k=1, \cdots, N$, and $G_{k k}$ are two-meson Green functions. $T_{j i}$ with $j=i(j \neq i)$ is the $T$-matrix element for elastic (inelastic) meson-meson scattering. Poles of the $T$-matrix elements are identified as resonances. ${ }^{1}$ The $T$-matrix elements yield phase shifts for elastic scattering and inelasticity for inelastic scattering. ${ }^{26}$ To generate 
all the resonances with isospin 0 and masses below $2 \mathrm{GeV}, S$-wave meson-meson scattering for total isospin $I=0$ and $1 / 2$ of the two mesons is studied in Ref.27 with 13 coupled channels.

A multi-channel generalization of the inverse amplitude method was given in Refs. ${ }^{[28}[29]$ where the $T$-matrix is a $N \times N$ matrix. Due to the unitarity the $T$-matrix is related to its inverse. Deriving the inverse from scattering amplitudes obtained in $\chi \mathrm{PT}$, the $T$-matrix elements acquire nonperturbative aspects which can produce resonances, elastic phase shifts, and inelasticity of inelastic meson-meson scattering.

In some decays and reactions where mesons are final states, final state interactions become appreciable and even important $[30 \mid 31$ To facilitate studies of these decays and reactions, experimental data on some meson-meson scattering amplitudes are parametrized ${ }^{32 \sqrt[34]{34}}$ For example, a study of $\pi \pi \rightarrow K \bar{K}$ scattering by means of partial-wave dispersion relations of the Roy-Steiner type is performed in Ref., 32 and precise parametrizations of the $S, P$, and $D$ partial waves in the $\pi \pi \rightarrow K \bar{K}$ scattering amplitude are obtained from the data.

\section{Inelastic Meson-Meson Scattering from Effective Meson Lagrangians}

Irrespective of chiral symmetry, effective meson Lagrangians with explicit resonances have been constructed to study meson-meson scattering. Let $\phi_{\mathrm{s}}, \phi_{\mathrm{p}}, \phi_{\mathrm{v}}^{\mu}$, and $\phi_{\mathrm{t}}^{\mu \nu}$ stand for the fields of the scalar meson, the pseudoscalar meson, the vector meson, and the tensor meson, respectively. For scattering between two pseudoscalar mesons interaction terms of the Lagrangian given in Ref. ${ }^{35}$ are

$$
\begin{gathered}
\mathcal{L}_{\mathrm{pps}}=\mathrm{g}_{\mathrm{pps}} m_{\mathrm{p}} \phi_{\mathrm{p}}(x) \phi_{\mathrm{p}}(x) \phi_{\mathrm{s}}(x), \\
\mathcal{L}_{\mathrm{ppv}}=\mathrm{g}_{\mathrm{ppv}} \phi_{\mathrm{p}}(x) \partial_{\mu} \phi_{\mathrm{p}}(x) \phi_{\mathrm{v}}^{\mu}(x), \\
\mathcal{L}_{\mathrm{ppt}}=\mathrm{g}_{\mathrm{ppt}} \frac{2}{m_{\pi}} \partial_{\mu} \phi_{\mathrm{p}}(x) \partial_{\nu} \phi_{\mathrm{p}}(x) \phi_{\mathrm{t}}^{\mu \nu}(x),
\end{gathered}
$$

where $m_{\mathrm{p}}\left(m_{\pi}\right)$ is the mass of the pseudoscalar meson (pion); $\mathrm{g}_{\mathrm{pps}}\left(\mathrm{g}_{\mathrm{ppv}}, \mathrm{g}_{\mathrm{ppt}}\right)$ is the coupling constant of the two pseudoscalar mesons and the scalar meson (vector meson, tensor meson). In scattering of two pseudoscalar mesons scalar mesons, vector mesons, and tensor mesons are propagators in the $s$ channel, the $t$ channel, and the $u$ channel. The $T$-matrix elements in the coupled-channel case are obtained from the Lippmann-Schwinger equations, and are used to get cross sections for mesonmeson reactions like $\pi^{+} \pi^{-} \rightarrow K \bar{K}$. With $\mathcal{L}_{\text {ppv }}$ isospin-averaged cross sections for $\pi \pi \rightarrow K \bar{K}, \rho \rho \rightarrow K \bar{K}, \pi \rho \rightarrow K \bar{K}^{*}$, and $\pi \rho \rightarrow K^{*} \bar{K}$ in vacuum have been obtained in Ref ${ }^{36}$ via exchange of either a kaon or a vector kaon between the two initial mesons. The cross sections depend on masses of initial and final mesons. The baryon-rich medium created in intermediate-energy nucleus-nucleus collisions modifies meson masses. Cross sections for the four reactions in medium may be obtained by using in-medium meson masses. 
Starting from a meson Lagrangian different from that in Ref., $\frac{35}{35} \pi K^{*} \rightarrow \rho K$, $\pi K^{*} \rightarrow \omega K, \pi K^{*} \rightarrow \phi K, \rho K^{*} \rightarrow \pi K, \omega K^{*} \rightarrow \pi K$, and $\phi K^{*} \rightarrow \pi K$ have been studied in Ref ${ }^{37}$ In calculations of cross sections for these reactions in vacuum the exchange of $K_{1}(1270)$ and $K_{2}^{*}(1430)$ in the $s$ channel, $h_{1}(1170)$ in the $t$ channel, etc. is taken into account.

\section{Meson-Meson Reactions due to Quark Interchange}

Meson $A$ is made of quark $q_{1}$ and antiquark $\bar{q}_{1}$, which may have different flavors, and meson $B$ consists of quark $q_{2}$ and antiquark $\bar{q}_{2}$, which may also have different flavors. In the collision of mesons $A$ and $B$ quark $q_{1}$ and quark $q_{2}$ interchange, and then $q_{2}$ and $\bar{q}_{1}$ combine to form meson $C$ and $q_{1}$ and $\bar{q}_{2}$ combine to form meson $D$. This is the quark interchange mechanism ${ }^{38}$ which leads to the scattering $A\left(q_{1} \bar{q}_{1}\right)+$ $B\left(q_{2} \bar{q}_{2}\right) \rightarrow C\left(q_{2} \bar{q}_{1}\right)+D\left(q_{1} \bar{q}_{2}\right)$. When an interaction takes place between constituents of mesons $A$ and $B$ prior to quark interchange, the scattering is designated as the prior form. When an interaction occurs between constituents of $q_{2} \bar{q}_{1}$ and $q_{1} \bar{q}_{2}$ after quark interchange, the scattering is designated as the post form. In the prior form of the scattering the color interaction between a constituent of meson $A$ and a constituent of meson $B$ turns the color-singlet states $A$ and $B$ into color-octet states of $q_{1} \bar{q}_{1}$ and $q_{2} \bar{q}_{2}$, respectively. During propagation of quarks and antiquarks interchange of $q_{1}$ and $q_{2}$ causes $q_{2}\left(q_{1}\right)$ to find $\bar{q}_{1}\left(\bar{q}_{2}\right)$ to get the color-singlet state $C(D)$. In the post form quark interchange produces a color-octet state of $q_{2} \bar{q}_{1}$ and another color-octet state of $q_{1} \bar{q}_{2}$. The color interaction between a constituent of $q_{2} \bar{q}_{1}$ and a constituent of $q_{1} \bar{q}_{2}$ makes $q_{2} \bar{q}_{1}\left(q_{1} \bar{q}_{2}\right)$ colorless so that the bound state $C(D)$ is formed.

The $S$-matrix element is 39

$$
S_{\mathrm{fi}}=\delta_{\mathrm{fi}}-\frac{(2 \pi)^{4} i \delta\left(E_{\mathrm{f}}-E_{\mathrm{i}}\right) \delta^{3}\left(\vec{P}_{\mathrm{f}}-\vec{P}_{\mathrm{i}}\right)}{V^{2} \sqrt{2 E_{A} 2 E_{B} 2 E_{C} 2 E_{D}}}\left(\mathcal{M}_{q_{1}}^{\text {prior }}+\mathcal{M}_{\bar{q}_{1} q_{2}}^{\text {prior }}+\mathcal{M}_{q_{1} q_{2}}^{\text {prior }}+\mathcal{M}_{\bar{q}_{1}}^{\text {prior }}\right),
$$

for scattering in the prior form or

$$
S_{\mathrm{fi}}=\delta_{\mathrm{fi}}-\frac{(2 \pi)^{4} i \delta\left(E_{\mathrm{f}}-E_{\mathrm{i}}\right) \delta^{3}\left(\vec{P}_{\mathrm{f}}-\vec{P}_{\mathrm{i}}\right)}{V^{2} \sqrt{2 E_{A} 2 E_{B} 2 E_{C} 2 E_{D}}}\left(\mathcal{M}_{q_{1} \bar{q}_{1}}^{\text {post }}+\mathcal{M}_{\bar{q}_{2} q_{2}}^{\text {post }}+\mathcal{M}_{q_{1} q_{2}}^{\text {post }}+\mathcal{M}_{\bar{q}_{1} \bar{q}_{2}}^{\text {post }}\right),
$$

for scattering in the post form, where $E_{j}$ is the energy of meson $j ; V$ is the volume in which every meson wave function is normalized; $E_{\mathrm{i}}$ and $\vec{P}_{\mathrm{i}}\left(E_{\mathrm{f}}\right.$ and $\left.\vec{P}_{\mathrm{f}}\right)$ are the total energy and the total three-dimensional momentum of the two initial (final) mesons, respectively; $\mathcal{M}_{q_{1} \bar{q}_{2}}^{\text {prior }}, \mathcal{M}_{\bar{q}_{1} q_{2}}^{\text {prior }}, \mathcal{M}_{q_{1} q_{2}}^{\text {prior }}, \mathcal{M}_{\bar{q}_{1} \bar{q}_{2}}^{\text {prior }}, \mathcal{M}_{q_{1} \bar{q}_{1}}^{\text {post }}, \mathcal{M}_{\bar{q}_{2} q_{2}}^{\text {post }}, \mathcal{M}_{q_{1} q_{2}}^{\text {post }}$, and $\mathcal{M}_{\bar{q}_{1} \bar{q}_{2}}^{\text {post }}$ are transition amplitudes. $\mathcal{M}_{q_{1} \bar{q}_{2}}^{\text {prior }}$ and $\mathcal{M}_{q_{1}}^{\text {post }}$ are given by

$$
\begin{aligned}
\mathcal{M}_{q_{1} \bar{q}_{2}}^{\text {prior }}= & \sqrt{2 E_{A} 2 E_{B} 2 E_{C} 2 E_{D}} \int \frac{d^{3} p_{q_{1} \bar{q}_{2}}}{(2 \pi)^{3}} \frac{d^{3} p_{q_{2} \bar{q}_{1}}}{(2 \pi)^{3}} \\
& \psi_{q_{1} \bar{q}_{2}}^{+}\left(\vec{p}_{q_{1} \bar{q}_{2}}\right) \psi_{q_{2} \bar{q}_{1}}^{+}\left(\vec{p}_{q_{2} \bar{q}_{1}}\right) V_{q_{1} \bar{q}_{2}} \psi_{q_{1} \bar{q}_{1}}\left(\vec{p}_{q_{1} \bar{q}_{1}}\right) \psi_{q_{2} \bar{q}_{2}}\left(\vec{p}_{q_{2} \bar{q}_{2}}\right),
\end{aligned}
$$




$$
\begin{aligned}
\mathcal{M}_{q_{1} \bar{q}_{1}}^{\text {post }}= & \sqrt{2 E_{A} 2 E_{B} 2 E_{C} 2 E_{D}} \int \frac{d^{3} p_{q_{1} \bar{q}_{1}}}{(2 \pi)^{3}} \frac{d^{3} p_{q_{2} \bar{q}_{2}}}{(2 \pi)^{3}} \\
& \psi_{q_{1} \bar{q}_{2}}^{+}\left(\vec{p}_{q_{1} \bar{q}_{2}}\right) \psi_{q_{2} \bar{q}_{1}}^{+}\left(\vec{p}_{q_{2} \bar{q}_{1}}\right) V_{q_{1} \bar{q}_{1}} \psi_{q_{1} \bar{q}_{1}}\left(\vec{p}_{q_{1} \bar{q}_{1}}\right) \psi_{q_{2} \bar{q}_{2}}\left(\vec{p}_{q_{2} \bar{q}_{2}}\right),
\end{aligned}
$$

where $\vec{p}_{a b}$ is the relative momentum of constituents $a$ and $b ; \psi_{a b}\left(\vec{p}_{a b}\right)$ is the mesonic quark-antiquark wave function which is the product of the color wave function, the flavor wave function, the spin wave function, and the relative-motion wave function of $a$ and $b ; \psi_{a b}^{+}$is the Hermitean conjugate of $\psi_{a b} . \mathcal{M}_{\bar{q}_{1} q_{2}}^{\text {prior }}\left(\mathcal{M}_{q_{1} q_{2}}^{\text {prior }}, \mathcal{M}_{\bar{q}_{1} \bar{q}_{2}}^{\text {prior }}\right)$ is obtained by replacing $V_{q_{1} \bar{q}_{2}}$ in Eq. (12) with $V_{\bar{q}_{1} q_{2}}\left(V_{q_{1} q_{2}}, V_{\bar{q}_{1} \bar{q}_{2}}\right)$, and $\mathcal{M}_{\bar{q}_{2} q_{2}}^{\text {post }}$ $\left(\mathcal{M}_{q_{1} q_{2}}^{\text {post }}, \mathcal{M}_{\bar{q}_{1} \bar{q}_{2}}^{\text {post }}\right)$ by replacing $V_{q_{1} \bar{q}_{1}}$ in Eq. (13) with $V_{\bar{q}_{2} q_{2}}\left(V_{q_{1} q_{2}}, V_{\bar{q}_{1} \bar{q}_{2}}\right) . V_{a b}$ is the potential between $a$ and $b$ in momentum space, and is the Fourier transform of the following expression in coordinate space $: \underline{40}$

$$
V_{a b}(\vec{r})=V_{\mathrm{si}}(\vec{r})+V_{\mathrm{sS}}(\vec{r}),
$$

where $\vec{r}$ is the relative coordinate of $a$ and $b, V_{\mathrm{si}}$ the central spin-independent potential, and $V_{\mathrm{ss}}$ the spin-spin interaction.

The spin-independent potential depends on temperature $T$ and below the critical temperature $T_{\mathrm{c}}=0.175 \mathrm{GeV}$ is given by

$$
V_{\mathrm{si}}(\vec{r})=-\frac{\vec{\lambda}_{a}}{2} \cdot \frac{\vec{\lambda}_{b}}{2} \xi_{1}\left[1.3-\left(\frac{T}{T_{\mathrm{c}}}\right)^{4}\right] \tanh \left(\xi_{2} r\right)+\frac{\vec{\lambda}_{a}}{2} \cdot \frac{\vec{\lambda}_{b}}{2} \frac{6 \pi}{25} \frac{v(\lambda r)}{r} \exp \left(-\xi_{3} r\right),
$$

where $\xi_{1}=0.525 \mathrm{GeV}, \xi_{2}=1.5\left[0.75+0.25\left(T / T_{\mathrm{c}}\right)^{10}\right]^{6} \mathrm{GeV}, \xi_{3}=0.6 \mathrm{GeV}$, and $\lambda=\sqrt{3 b_{0} / 16 \pi^{2} \alpha^{\prime}}$ in which $\alpha^{\prime}=1.04 \mathrm{GeV}^{-2}$ and $b_{0}=11-\frac{2}{3} N_{f}$ with the quark flavor number $N_{f}=4$. $\vec{\lambda}_{a}$ are the Gell-Mann matrices for the color generators of constituent quark or antiquark labeled as $a$. The dimensionless function $v(x)$ is given by Buchmüller and Tye ${ }^{41}$ The short-distance part of the spin-independent potential originates from one-gluon exchange plus perturbative one- and two-loop corrections. The intermediate-distance and large-distance part of the spin-independent potential fits well the numerical potential which was obtained in the lattice gauge calculations $\stackrel{42}{42}$ At large distances the spin-independent potential is independent of the relative coordinate and obviously exhibits a plateau at $T / T_{\mathrm{c}}>0.55$. The plateau height decreases with increasing temperature. This means that confinement becomes weaker and weaker.

Denote the spin and the mass of constituent $a$ by $\vec{s}_{a}$ and $m_{a}$, respectively. The spin-spin interaction with relativistic effects $\frac{38 \mid 43}{44}$ is

$$
V_{\mathrm{ss}}(\vec{r})=-\frac{\vec{\lambda}_{a}}{2} \cdot \frac{\vec{\lambda}_{b}}{2} \frac{16 \pi^{2}}{25} \frac{d^{3}}{\pi^{3 / 2}} \mathrm{e}^{-d^{2} r^{2}} \frac{\vec{s}_{a} \cdot \vec{s}_{b}}{m_{a} m_{b}}+\frac{\vec{\lambda}_{a}}{2} \cdot \frac{\vec{\lambda}_{b}}{2} \frac{4 \pi}{25} \frac{1}{r} \frac{d^{2} v(\lambda r)}{d r^{2}} \frac{\vec{s}_{a} \cdot \vec{s}_{b}}{m_{a} m_{b}},
$$

with

$$
d^{2}=d_{1}^{2}\left[\frac{1}{2}+\frac{1}{2}\left(\frac{4 m_{a} m_{b}}{\left(m_{a}+m_{b}\right)^{2}}\right)^{4}\right]+d_{2}^{2}\left(\frac{2 m_{a} m_{b}}{m_{a}+m_{b}}\right)^{2},
$$

where $d_{1}=0.15 \mathrm{GeV}$ and $d_{2}=0.705$. 
$V_{a b}(\vec{r})$ at $T=0$ provides a quark potential in vacuum, and for $T>0.6 T_{\mathrm{c}}$ does so in hadronic matter that results from the quark-gluon plasma. Meson masses and mesonic quark-antiquark relative-motion wave functions in coordinate space are determined from the Schrödinger equation with $V_{a b}(\vec{r})$. They depend on temperature. The first term given in Eq. (15) stands for the confining potential. In the confinement regime the mesonic quark-antiquark relative-motion wave functions mainly determined by the confining potential are nonperturbative. At low energies near threshold of $A\left(q_{1} \bar{q}_{1}\right)+B\left(q_{2} \bar{q}_{2}\right) \rightarrow C\left(q_{2} \bar{q}_{1}\right)+D\left(q_{1} \bar{q}_{2}\right)$ the nonperturbative part of the $q_{1} \bar{q}_{1}$ and $q_{2} \bar{q}_{2}$ wave functions must overlap. Even though the distance of $q_{2}$ and $\bar{q}_{1}\left(q_{1}\right.$ and $\left.\bar{q}_{2}\right)$ is large, the nonperturbative correlation corresponding to the nonperturbative part leads to formation of a bound state of $q_{2}$ and $\bar{q}_{1}\left(q_{1}\right.$ and $\left.\bar{q}_{2}\right)$ during the hadronization of $q_{1}, \bar{q}_{1}, q_{2}$, and $\bar{q}_{2}$, i.e., meson $C(D)$.

Since the sum of the spin operators of $q_{1}, \bar{q}_{1}, q_{2}$, and $\bar{q}_{2}$ commutes with the potential $V_{a b}$, the total spin of the two final mesons equals the one of the two initial mesons. The following reactions have been considered in Ref.. $\frac{\sqrt[45]{ }}{4}$

$$
\begin{array}{r}
I=2 \pi \pi \rightarrow \rho \rho, \quad I=1 K K \rightarrow K^{*} K^{*}, \quad I=1 K K^{*} \rightarrow K^{*} K^{*}, \\
I=3 / 2 \pi K \rightarrow \rho K^{*}, \quad I=3 / 2 \pi K^{*} \rightarrow \rho K^{*}, \\
I=3 / 2 \rho K \rightarrow \rho K^{*}, \quad I=3 / 2 \pi K^{*} \rightarrow \rho K .
\end{array}
$$

The seven isospin channels of these reactions are endothermic. The unpolarized cross section is the average of the one for scattering in the prior form and the one for scattering in the post form. Denote by $\sqrt{s}$ the center-of-mass energy of the two initial mesons. While $\sqrt{s}$ increases from threshold, the cross section for each isospin channel rises very rapidly from 0 , arrives at a maximum value (peak cross section), and decreases rapidly. The cross sections for these reactions change considerably with the temperature of hadronic matter. For example, every reaction has a rising peak cross section when the temperature becomes critical.

\section{Meson-Meson Reactions due to Quark-Antiquark Annihilation and Creation}

A quark in an initial meson and an antiquark in another initial meson annihilate into a gluon, and subsequently the gluon creates another quark-antiquark pair. This quark-antiquark annihilation and creation causes two-to-two meson-meson scattering, $A\left(q_{1} \bar{q}_{1}\right)+B\left(q_{2} \bar{q}_{2}\right) \rightarrow C\left(q_{3} \bar{q}_{1}\right)+D\left(q_{2} \bar{q}_{4}\right)$ from $q_{1}+\bar{q}_{2} \rightarrow q_{3}+\bar{q}_{4}$ and $A\left(q_{1} \bar{q}_{1}\right)+B\left(q_{2} \bar{q}_{2}\right) \rightarrow C\left(q_{1} \bar{q}_{4}\right)+D\left(q_{3} \bar{q}_{2}\right)$ from $\bar{q}_{1}+q_{2} \rightarrow q_{3}+\bar{q}_{4}$. The $S$-matrix element for $A+B \rightarrow C+D$ is $\underline{46}$

$$
S_{\mathrm{fi}}=\delta_{\mathrm{fi}}-(2 \pi)^{4} i \delta\left(E_{\mathrm{f}}-E_{\mathrm{i}}\right) \delta^{3}\left(\vec{P}_{\mathrm{f}}-\vec{P}_{\mathrm{i}}\right) \frac{\mathcal{M}_{\mathrm{a} q_{1} \bar{q}_{2}}+\mathcal{M}_{\mathrm{a} \bar{q}_{1} q_{2}}}{V^{2} \sqrt{2 E_{A} 2 E_{B} 2 E_{C} 2 E_{D}}},
$$

where $\mathcal{M}_{\mathrm{a} q_{1} \bar{q}_{2}}$ and $\mathcal{M}_{\mathrm{a} \bar{q}_{1} q_{2}}$ are the transition amplitudes that may be written as:

$$
\begin{aligned}
\mathcal{M}_{\mathrm{a} q_{1} \bar{q}_{2}}= & \sqrt{2 E_{A} 2 E_{B} 2 E_{C} 2 E_{D}} \int \frac{d^{3} p_{q_{1} \bar{q}_{1}}}{(2 \pi)^{3}} \frac{d^{3} p_{q_{2} \bar{q}_{2}}}{(2 \pi)^{3}} \\
& \psi_{q_{3} \bar{q}_{1}}^{+}\left(\vec{p}_{q_{3} \bar{q}_{1}}\right) \psi_{q_{2} \bar{q}_{4}}^{+}\left(\vec{p}_{q_{2} \bar{q}_{4}}\right) V_{\mathrm{a}_{1} \bar{q}_{2}} \psi_{q_{1} \bar{q}_{1}}\left(\vec{p}_{q_{1} \bar{q}_{1}}\right) \psi_{q_{2} \bar{q}_{2}}\left(\vec{p}_{q_{2} \bar{q}_{2}}\right),
\end{aligned}
$$




$$
\begin{aligned}
\mathcal{M}_{\mathrm{a} \bar{q}_{1} q_{2}}= & \sqrt{2 E_{A} 2 E_{B} 2 E_{C} 2 E_{D}} \int \frac{d^{3} p_{q_{1} \bar{q}_{1}}}{(2 \pi)^{3}} \frac{d^{3} p_{q_{2} \bar{q}_{2}}}{(2 \pi)^{3}} \\
& \psi_{q_{1} \bar{q}_{4}}^{+}\left(\vec{p}_{q_{1} \bar{q}_{4}}\right) \psi_{q_{3} \bar{q}_{2}}^{+}\left(\vec{p}_{q_{3} \bar{q}_{2}}\right) V_{\mathrm{a} \bar{q}_{1} q_{2}} \psi_{q_{1} \bar{q}_{1}}\left(\vec{p}_{q_{1} \bar{q}_{1}}\right) \psi_{q_{2} \bar{q}_{2}}\left(\vec{p}_{q_{2} \bar{q}_{2}}\right),
\end{aligned}
$$

where $V_{\mathrm{a} q_{1} \bar{q}_{2}}$ and $V_{\mathrm{a} \bar{q}_{1} q_{2}}$ are the transition potentials for $q_{1}+\bar{q}_{2} \rightarrow q_{3}+\bar{q}_{4}$ and $\bar{q}_{1}+q_{2} \rightarrow q_{3}+\bar{q}_{4}$, respectively. The transition potentials have been derived in perturbative quantum chromodynamics (QCD). Since the commutators of the transition potentials and the sum of the four constituent spin operators do not equal zero, the total spin of the two final mesons may not equal the one of the two initial mesons 47 The following reactions have been considered in Refs.46] 47

$$
\begin{array}{r}
\pi \pi \rightarrow K \bar{K}, \quad \pi \pi \rightarrow K \bar{K}^{*}, \quad \pi \pi \rightarrow K^{*} \bar{K}, \quad \pi \pi \rightarrow K^{*} \bar{K}^{*}, \\
\pi \rho \rightarrow K \bar{K}, \quad \pi \rho \rightarrow K \bar{K}^{*}, \quad \pi \rho \rightarrow K^{*} \bar{K}, \quad \pi \rho \rightarrow K^{*} \bar{K}^{*}, \\
\rho \rho \rightarrow K^{*} \bar{K}^{*}, \quad K \bar{K} \rightarrow \rho \rho, \quad K \bar{K}^{*} \rightarrow \rho \rho, \quad K^{*} \bar{K} \rightarrow \rho \rho, \\
K \bar{K} \rightarrow K \bar{K}^{*}, \quad K \bar{K} \rightarrow K^{*} \bar{K}, \quad K \bar{K} \rightarrow K^{*} \bar{K}^{*}, \\
K \bar{K}^{*} \rightarrow K^{*} \bar{K}^{*}, \quad K^{*} \bar{K} \rightarrow K^{*} \bar{K}^{*}, \\
\pi K \rightarrow \pi K^{*}, \quad \pi K \rightarrow \rho K, \\
I=1 \pi \pi \rightarrow \rho \rho .
\end{array}
$$

In some regimes these reactions receive contributions from quark-antiquark annihilation and creation. Unpolarized cross sections for these reactions depend on $T$ and $\sqrt{s}$. The temperature dependence arises from the meson masses and the quark-antiquark relative-motion wave functions of the initial and final mesons. With increasing $\sqrt{s}$ from the threshold energy, the cross section for any endothermic reaction of these reactions at a given temperature increases very rapidly to a peak cross section first, and then decreases or displays a plateau for some energy interval.

\section{Meson-Meson Reactions due to Quark-Interchange as well as Quark-Antiquark Annihilation and Creation}

Transition amplitudes are proportional to flavor matrix elements. Flavor matrix elements are calculated with flavor wave functions of initial and final mesons. If the flavor matrix element of a reaction is zero, the reaction can not occur. The flavor matrix element depends on the total isospin of the two initial or final mesons and the mechanism (quark interchange, quark-antiquark annihilation and creation) which causes the reaction. From the flavor matrix elements of $\pi \pi \rightarrow \rho \rho$ we know that $\pi \pi \rightarrow \rho \rho$ for $I=2$ is governed by quark interchange, $\pi \pi \rightarrow \rho \rho$ for $I=1$ by quark-antiquark annihilation and creation, and $\pi \pi \rightarrow \rho \rho$ for $I=0$ by quark interchange as well as quark-antiquark annihilation and creation. Even though the four reactions $\pi K \rightarrow \rho K^{*}, \pi K^{*} \rightarrow \rho K, \pi K^{*} \rightarrow \rho K^{*}$, and $\rho K \rightarrow \rho K^{*}$ are governed by quark interchange when $I=3 / 2$, they are governed by quark interchange as well as quark-antiquark annihilation and creation when $I=1 / 2$. Therefore, some isospin channels of reactions are governed by quark interchange as well as quark-antiquark 
annihilation and creation. The $S$-matrix element for this kind of scattering is $\underline{46}$

$$
\begin{aligned}
S_{\mathrm{fi}}= & \delta_{\mathrm{fi}}-\frac{(2 \pi)^{4} i \delta\left(E_{\mathrm{f}}-E_{\mathrm{i}}\right) \delta^{3}\left(\vec{P}_{\mathrm{f}}-\vec{P}_{\mathrm{i}}\right)}{V^{2} \sqrt{2 E_{A} 2 E_{B} 2 E_{C} 2 E_{D}}} \\
& \left(\mathcal{M}_{q_{1} \bar{q}_{2}}^{\text {prior }}+\mathcal{M}_{\bar{q}_{1} q_{2}}^{\text {prior }}+\mathcal{M}_{q_{1} q_{2}}^{\text {prior }}+\mathcal{M}_{\bar{q}_{1} \bar{q}_{2}}^{\text {prior }}+\mathcal{M}_{\mathrm{a} q_{1} \bar{q}_{2}}+\mathcal{M}_{\mathrm{a} \bar{q}_{1} q_{2}}\right),
\end{aligned}
$$

with the quark-interchange process in the prior form or

$$
\begin{aligned}
S_{\mathrm{fi}}= & \delta_{\mathrm{fi}}-\frac{(2 \pi)^{4} i \delta\left(E_{\mathrm{f}}-E_{\mathrm{i}}\right) \delta^{3}\left(\vec{P}_{\mathrm{f}}-\vec{P}_{\mathrm{i}}\right)}{V^{2} \sqrt{2 E_{A} 2 E_{B} 2 E_{C} 2 E_{D}}} \\
& \left(\mathcal{M}_{q_{1} \bar{q}_{1}}^{\text {post }}+\mathcal{M}_{\bar{q}_{2} q_{2}}^{\text {post }}+\mathcal{M}_{q_{1} q_{2}}^{\text {post }}+\mathcal{M}_{\bar{q}_{1} \bar{q}_{2}}^{\text {post }}+\mathcal{M}_{\mathrm{a} q_{1} \bar{q}_{2}}+\mathcal{M}_{\mathrm{a} \bar{q}_{1} q_{2}}\right),
\end{aligned}
$$

with the quark-interchange process in the post form.

Unpolarized cross sections for $\pi \pi \rightarrow \rho \rho$ for $I=0$ have been obtained in Ref. ${ }^{46}$ and ones for $\pi K \rightarrow \rho K^{*}$ for $I=1 / 2, \pi K^{*} \rightarrow \rho K$ for $I=1 / 2, \pi K^{*} \rightarrow \rho K^{*}$ for $I=1 / 2$, and $\rho K \rightarrow \rho K^{*}$ for $I=1 / 2$ have been obtained in Ref. ${ }^{48}$ Near threshold quark interchange dominates the five channels near the critical temperature; in the other energy region quark-antiquark annihilation and creation may dominate the five channels.

\section{Meson-Meson Reactions from Quark-Antiquark Annihilation}

A quark in one initial meson and an antiquark in the other initial meson annihilate into a gluon, and subsequently the gluon is absorbed by the other antiquark or quark. This quark-antiquark annihilation leads to two-to-one meson-meson scattering. The reaction $A\left(q_{1} \bar{q}_{1}\right)+B\left(q_{2} \bar{q}_{2}\right) \rightarrow H\left(q_{2} \bar{q}_{1}\right)$ is caused by $q_{1}+\bar{q}_{2}+\bar{q}_{1} \rightarrow \bar{q}_{1}$ and $q_{1}+\bar{q}_{2}+q_{2} \rightarrow q_{2}$, and $A\left(q_{1} \bar{q}_{1}\right)+B\left(q_{2} \bar{q}_{2}\right) \rightarrow H\left(q_{1} \bar{q}_{2}\right)$ is caused by $q_{2}+\bar{q}_{1}+q_{1} \rightarrow q_{1}$ and $q_{2}+\bar{q}_{1}+\bar{q}_{2} \rightarrow \bar{q}_{2}$. The $S$-matrix element for $A+B \rightarrow H$ is $\underline{48}$

$$
S_{\mathrm{fi}}=\delta_{\mathrm{fi}}-(2 \pi)^{4} i \delta\left(E_{\mathrm{f}}-E_{\mathrm{i}}\right) \delta^{3}\left(\vec{P}_{\mathrm{f}}-\vec{P}_{\mathrm{i}}\right) \frac{\mathcal{M}_{\mathrm{r} q_{1} \bar{q}_{2} \bar{q}_{1}}+\mathcal{M}_{\mathrm{r} q_{1} \bar{q}_{2} q_{2}}+\mathcal{M}_{\mathrm{r} q_{2} \bar{q}_{1} q_{1}}+\mathcal{M}_{\mathrm{r} q_{2} \bar{q}_{1} \bar{q}_{2}}}{V^{\frac{3}{2}} \sqrt{2 E_{A} 2 E_{B} 2 E_{H}}},
$$

where $\mathcal{M}_{\mathrm{r} q_{1} \bar{q}_{2} \bar{q}_{1}}, \mathcal{M}_{\mathrm{r} q_{1} \bar{q}_{2} q_{2}}, \mathcal{M}_{\mathrm{r} q_{2} \bar{q}_{1} q_{1}}$, and $\mathcal{M}_{\mathrm{r} q_{2} \bar{q}_{1} \bar{q}_{2}}$ are the transition amplitudes that may be written as

$$
\begin{aligned}
\mathcal{M}_{\mathrm{r} q_{1} \bar{q}_{2} \bar{q}_{1}}= & \sqrt{2 E_{A} 2 E_{B} 2 E_{H}} \int \frac{d^{3} p_{q_{1}} \bar{q}_{1}}{(2 \pi)^{3}} \frac{d^{3} p_{q_{2} \bar{q}_{2}}}{(2 \pi)^{3}} \psi_{q_{2} \bar{q}_{1}}^{+}\left(\vec{p}_{q_{2} \bar{q}_{1}}\right) V_{\mathrm{r} q_{1} \bar{q}_{2} \bar{q}_{1}} \\
& \psi_{q_{1} \bar{q}_{1}}\left(\vec{p}_{q_{1} \bar{q}_{1}}\right) \psi_{q_{2} \bar{q}_{2}}\left(\vec{p}_{q_{2} \bar{q}_{2}}\right), \\
\mathcal{M}_{\mathrm{r} q_{1} \bar{q}_{2} q_{2}}= & \sqrt{2 E_{A} 2 E_{B} 2 E_{H}} \int \frac{d^{3} p_{q_{1}} \bar{q}_{1}}{(2 \pi)^{3}} \frac{d^{3} p_{q_{2} \bar{q}_{2}}}{(2 \pi)^{3}} \psi_{q_{2} \bar{q}_{1}}^{+}\left(\vec{p}_{q_{2} \bar{q}_{1}}\right) V_{\mathrm{r} q_{1} \bar{q}_{2} q_{2}} \\
& \psi_{q_{1} \bar{q}_{1}}\left(\vec{p}_{q_{1} \bar{q}_{1}}\right) \psi_{q_{2} \bar{q}_{2}}\left(\vec{p}_{q_{2} \bar{q}_{2}}\right), \\
\mathcal{M}_{\mathrm{r} q_{2} \bar{q}_{1} q_{1}}= & \sqrt{2 E_{A} 2 E_{B} 2 E_{H}} \int \frac{d^{3} p_{q_{1}} \bar{q}_{1}}{(2 \pi)^{3}} \frac{d^{3} p_{q_{2} \bar{q}_{2}}}{(2 \pi)^{3}} \psi_{q_{1} \bar{q}_{2}}^{+}\left(\vec{p}_{q_{1} \bar{q}_{2}}\right) V_{\mathrm{r} q_{2} \bar{q}_{1} q_{1}} \\
& \psi_{q_{1} \bar{q}_{1}}\left(\vec{p}_{q_{1} \bar{q}_{1}}\right) \psi_{q_{2} \bar{q}_{2}}\left(\vec{p}_{q_{2} \bar{q}_{2}}\right),
\end{aligned}
$$




$$
\begin{aligned}
\mathcal{M}_{\mathrm{r} q_{2} \bar{q}_{1} \bar{q}_{2}}= & \sqrt{2 E_{A} 2 E_{B} 2 E_{H}} \int \frac{d^{3} p_{q_{1}} \bar{q}_{1}}{(2 \pi)^{3}} \frac{d^{3} p_{q_{2} \bar{q}_{2}}}{(2 \pi)^{3}} \psi_{q_{1} \bar{q}_{2}}^{+}\left(\vec{p}_{q_{1} \bar{q}_{2}}\right) V_{\mathrm{r} q_{2} \bar{q}_{1} \bar{q}_{2}} \\
& \psi_{q_{1} \bar{q}_{1}}\left(\vec{p}_{q_{1} \bar{q}_{1}}\right) \psi_{q_{2} \bar{q}_{2}}\left(\vec{p}_{q_{2} \bar{q}_{2}}\right),
\end{aligned}
$$

where $V_{\mathrm{r} q_{1} \bar{q}_{2} \bar{q}_{1}}\left(V_{\mathrm{r} q_{1} \bar{q}_{2} q_{2}}\right)$ represents the transition potential for the annihilation of $q_{1}$ and $\bar{q}_{2}$ into a gluon and the subsequent absorption of the gluon by $\bar{q}_{1}$ in meson $A$ ( $q_{2}$ in meson $\left.B\right) ; V_{\mathrm{r} q_{2} \bar{q}_{1} q_{1}}\left(V_{\mathrm{r} q_{2} \bar{q}_{1} \bar{q}_{2}}\right)$ represents the transition potential for the annihilation of $q_{2}$ and $\bar{q}_{1}$ into a gluon and the subsequent absorption of the gluon by $q_{1}$ in meson $A\left(\bar{q}_{2}\right.$ in meson $\left.B\right)$. The transition potentials have been derived from the Feynman rules in perturbative QCD.

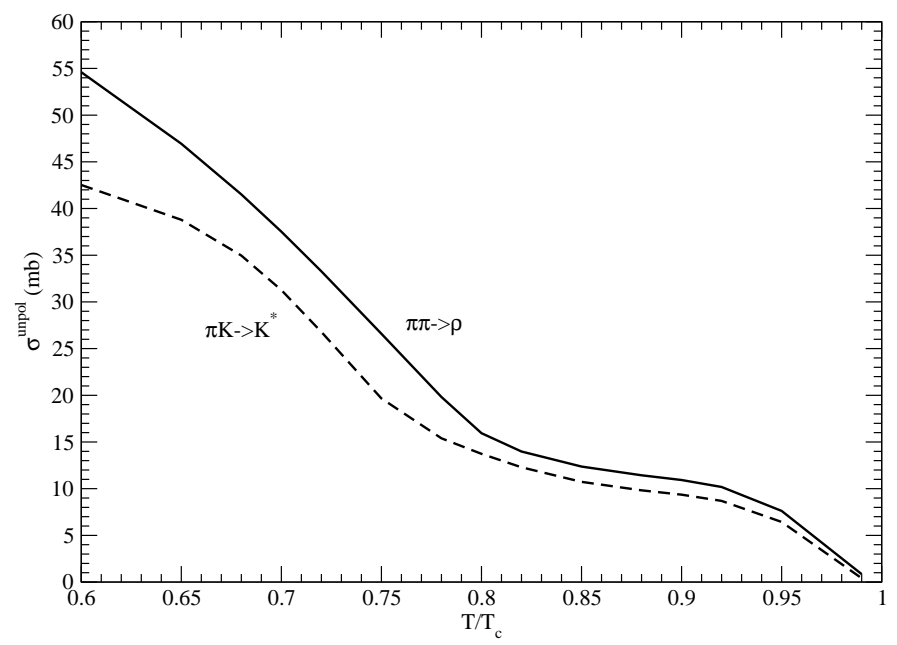

Fig. 1. Unpolarized cross sections for $\pi \pi \rightarrow \rho$ and $\pi K \rightarrow K^{*}$ as functions of $T / T_{\mathrm{c}}$.

The transition amplitudes result in cross sections for $\pi \pi \rightarrow \rho$ and $\pi K \rightarrow K^{*}$

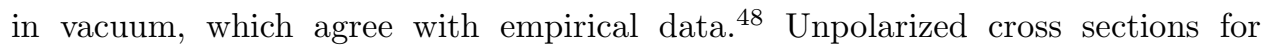
$0.6 \leq T / T_{\mathrm{c}}<1$ are shown in Fig. 1. The weakening confinement with increasing temperature makes combining the final quark and the final antiquark into a meson more difficult, and thus reduces the cross sections. The temperature dependence of the cross sections is remarkable.

\section{Meson-Meson Reactions from Quark-Antiquark Creation}

In the collision of mesons $A\left(q_{1} \bar{q}_{1}\right)$ and $B\left(q_{2} \bar{q}_{2}\right)$ a constituent quark or antiquark may emit a virtual gluon which subsequently splits into quark $q_{3}$ and antiquark $\bar{q}_{4}$. If $\bar{q}_{4}$ and $q_{1}$ combine into a meson, then $q_{3}$ and $\bar{q}_{2}$ combine into another meson. If $\bar{q}_{4}$ and $q_{2}$ combine into a meson, then $q_{3}$ and $\bar{q}_{1}$ combine into another meson. The 
quark-antiquark creation thus leads to the reaction $A\left(q_{1} \bar{q}_{1}\right)+B\left(q_{2} \bar{q}_{2}\right) \rightarrow C_{1}\left(q_{1} \bar{q}_{4}\right)+$ $C_{2}\left(q_{2} \bar{q}_{1}\right)+C_{3}\left(q_{3} \bar{q}_{2}\right)$ and $A\left(q_{1} \bar{q}_{1}\right)+B\left(q_{2} \bar{q}_{2}\right) \rightarrow C_{1}\left(q_{1} \bar{q}_{2}\right)+C_{2}\left(q_{2} \bar{q}_{4}\right)+C_{3}\left(q_{3} \bar{q}_{1}\right)$. The $S$-matrix element for $A+B \rightarrow C_{1}+C_{2}+C_{3}$ is $\frac{16}{16}$

$$
\begin{aligned}
S_{\mathrm{fi}}= & \delta_{\mathrm{fi}}-\frac{(2 \pi)^{4} i \delta\left(E_{\mathrm{f}}-E_{\mathrm{i}}\right) \delta^{3}\left(\vec{P}_{\mathrm{f}}-\vec{P}_{\mathrm{i}}\right)}{\sqrt{V^{5}} \sqrt{2 E_{A} 2 E_{B} 2 E_{C_{1}} 2 E_{C_{2}} 2 E_{C_{3}}}} \\
& \left(\mathcal{M}_{\mathrm{D}_{1}}+\mathcal{M}_{\mathrm{D}_{2}}+\mathcal{M}_{\mathrm{D}_{3}}+\mathcal{M}_{\mathrm{D}_{4}}+\mathcal{M}_{\mathrm{D}_{5}}+\mathcal{M}_{\mathrm{D}_{6}}+\mathcal{M}_{\mathrm{D}_{7}}+\mathcal{M}_{\mathrm{D}_{8}}\right),
\end{aligned}
$$

where $\mathcal{M}_{\mathrm{D}_{h}}(h=1, \cdots, 8)$ are transition amplitudes. $\mathcal{M}_{\mathrm{D}_{1}}$ and $\mathcal{M}_{\mathrm{D}_{5}}$ may be written as

$$
\begin{aligned}
\mathcal{M}_{\mathrm{D}_{1}}= & \sqrt{2 E_{A} 2 E_{B} 2 E_{C_{1}} 2 E_{C_{2}} 2 E_{C_{3}}} \int \frac{d^{3} p_{q_{2} \bar{q}_{2}}}{(2 \pi)^{3}} \frac{d^{3} p_{q_{1} \bar{q}_{4}}}{(2 \pi)^{3}} \\
& \psi_{q_{1} \bar{q}_{4}}^{+}\left(\vec{p}_{q_{1} \bar{q}_{4}}\right) \psi_{q_{2} \bar{q}_{1}}^{+}\left(\vec{p}_{q_{2} \bar{q}_{1}}\right) \psi_{q_{3} \bar{q}_{2}}^{+}\left(\vec{p}_{q_{3} \bar{q}_{2}}\right) V_{\mathrm{D}_{1}} \psi_{q_{1} \bar{q}_{1}}\left(\vec{p}_{q_{1} \bar{q}_{1}}\right) \psi_{q_{2} \bar{q}_{2}}\left(\vec{p}_{q_{2} \bar{q}_{2}}\right), \\
\mathcal{M}_{\mathrm{D}_{5}=} & \sqrt{2 E_{A} 2 E_{B} 2 E_{C_{1}} 2 E_{C_{2}} 2 E_{C_{3}}} \int \frac{d^{3} p_{q_{1}} \bar{q}_{1}}{(2 \pi)^{3}} \frac{d^{3} p_{q_{2} \bar{q}_{2}}}{(2 \pi)^{3}} \\
& \psi_{q_{1} \bar{q}_{2}}^{+}\left(\vec{p}_{q_{1} \bar{q}_{2}}\right) \psi_{q_{2} \bar{q}_{4}}^{+}\left(\vec{p}_{q_{2} \bar{q}_{4}}\right) \psi_{q_{3} \bar{q}_{1}}^{+}\left(\vec{p}_{q_{3} \bar{q}_{1}}\right) V_{\mathrm{D}_{5}} \psi_{q_{1} \bar{q}_{1}}\left(\vec{p}_{q_{1} \bar{q}_{1}}\right) \psi_{q_{2} \bar{q}_{2}}\left(\vec{p}_{q_{2} \bar{q}_{2}}\right) .
\end{aligned}
$$

$\mathcal{M}_{\mathrm{D}_{2}}\left(\mathcal{M}_{\mathrm{D}_{3}}, \mathcal{M}_{\mathrm{D}_{4}}\right)$ is obtained by replacing $d^{3} p_{q_{2} \bar{q}_{2}} d^{3} p_{q_{1} \bar{q}_{4}} V_{\mathrm{D}_{1}}$ in Eq. (29) with $d^{3} p_{q_{1} \bar{q}_{1}} d^{3} p_{q_{2} \bar{q}_{2}} V_{\mathrm{D}_{2}}\left(d^{3} p_{q_{1} \bar{q}_{1}} d^{3} p_{q_{2} \bar{q}_{2}} V_{\mathrm{D}_{3}}, d^{3} p_{q_{1} \bar{q}_{1}} d^{3} p_{q_{3} \bar{q}_{2}} V_{\mathrm{D}_{4}}\right)$, and $\mathcal{M}_{\mathrm{D}_{6}}\left(\mathcal{M}_{\mathrm{D}_{7}}\right.$, $\left.\mathcal{M}_{\mathrm{D}_{8}}\right)$ by replacing $d^{3} p_{q_{1} \bar{q}_{1}} d^{3} p_{q_{2} \bar{q}_{2}} V_{\mathrm{D}_{5}}$ in Eq. (30) with $d^{3} p_{q_{2} \bar{q}_{2}} d^{3} p_{q_{3} \bar{q}_{1}} V_{\mathrm{D}_{6}}$ $\left(d^{3} p_{q_{1} \bar{q}_{1}} d^{3} p_{q_{2} \bar{q}_{4}} V_{\mathrm{D}_{7}}, d^{3} p_{q_{1} \bar{q}_{1}} d^{3} p_{q_{2} \bar{q}_{2}} V_{\mathrm{D}_{8}}\right) . V_{\mathrm{D}_{1}}\left(V_{\mathrm{D}_{2}}, V_{\mathrm{D}_{3}}, V_{\mathrm{D}_{4}}\right)$ represents the transition potential for $q_{1} \rightarrow q_{1}+q_{3}+\bar{q}_{4}\left(\bar{q}_{1} \rightarrow \bar{q}_{1}+q_{3}+\bar{q}_{4}, q_{2} \rightarrow q_{2}+q_{3}+\bar{q}_{4}\right.$, $\left.\bar{q}_{2} \rightarrow \bar{q}_{2}+q_{3}+\bar{q}_{4}\right)$ in $A\left(q_{1} \bar{q}_{1}\right)+B\left(q_{2} \bar{q}_{2}\right) \rightarrow C_{1}\left(q_{1} \bar{q}_{4}\right)+C_{2}\left(q_{2} \bar{q}_{1}\right)+C_{3}\left(q_{3} \bar{q}_{2}\right)$, and $V_{\mathrm{D}_{5}}\left(V_{\mathrm{D}_{6}}, V_{\mathrm{D}_{7}}, V_{\mathrm{D}_{8}}\right)$ indicates the transition potential for $q_{1} \rightarrow q_{1}+q_{3}+\bar{q}_{4}$ $\left(\bar{q}_{1} \rightarrow \bar{q}_{1}+q_{3}+\bar{q}_{4}, q_{2} \rightarrow q_{2}+q_{3}+\bar{q}_{4}, \bar{q}_{2} \rightarrow \bar{q}_{2}+q_{3}+\bar{q}_{4}\right)$ in $A\left(q_{1} \bar{q}_{1}\right)+B\left(q_{2} \bar{q}_{2}\right) \rightarrow$ $C_{1}\left(q_{1} \bar{q}_{2}\right)+C_{2}\left(q_{2} \bar{q}_{4}\right)+C_{3}\left(q_{3} \bar{q}_{1}\right)$. The transition potentials have been derived in perturbative QCD.

The following two-to-three meson-meson reactions are considered in Ref.!16

$$
\pi \pi \rightarrow \pi K \bar{K}, \pi K \rightarrow \pi \pi K, \pi K \rightarrow K K \bar{K}, K K \rightarrow \pi K K, K \bar{K} \rightarrow \pi K \bar{K} .
$$

Unpolarized cross sections for these reactions have been obtained from the eight transition amplitudes. At zero temperature the cross sections for $\pi K \rightarrow \pi \pi K$ for $I=3 / 2$ are near the experimental data. If the cross section for any reaction at a given temperature has a maximum, then the peak cross section decreases as the temperature approaches the critical temperature. By comparison with inelastic twoto-two meson-meson scattering, we find that two-to-three meson-meson scattering may be as important as inelastic two-to-two meson-meson scattering.

\section{Multi Mesons produced in Meson-Meson Reactions}

Meson-meson scattering is usually governed by a single Feynman diagram each, i.e. one transition amplitude corresponds to one Feynman diagram at tree level. Denote inelastic meson-meson scattering by $A\left(q_{1} \bar{q}_{1}\right)+B\left(q_{2} \bar{q}_{2}\right) \rightarrow C_{1}\left(q_{1}^{\prime} \bar{q}_{1}^{\prime}\right)+\cdots+C_{\mathrm{n}}\left(q_{\mathrm{n}}^{\prime} \bar{q}_{\mathrm{n}}^{\prime}\right)$, 
and let $\mathrm{D}_{1}, \cdots, \mathrm{D}_{\mathrm{m}}$ stand for the Feynman diagrams. For diagram $\mathrm{D}_{i}$ the transition amplitude is written as

$$
\begin{aligned}
\mathcal{M}_{\mathrm{D}_{i}}= & \sqrt{2 E_{A} 2 E_{B} 2 E_{C_{1}} \cdots 2 E_{C_{\mathrm{n}}}} \int \frac{d^{3} p_{a \bar{b}}}{(2 \pi)^{3}} \frac{d^{3} p_{c \bar{d}}}{(2 \pi)^{3}} \\
& \psi_{q_{1}^{\prime} \bar{q}_{1}^{\prime}}^{+}\left(\vec{p}_{q_{1}^{\prime} \bar{q}_{1}^{\prime}}\right) \cdots \psi_{q_{\mathrm{n}}^{\prime} \bar{q}_{\mathrm{n}}^{\prime}}^{+}\left(\vec{p}_{q_{\mathrm{n}}^{\prime} \bar{q}_{\mathrm{n}}^{\prime}}\right) V_{\mathrm{D}_{i}} \psi_{q_{1} \bar{q}_{1}}\left(\vec{p}_{q_{1} \bar{q}_{1}}\right) \psi_{q_{2} \bar{q}_{2}}\left(\vec{p}_{q_{2} \bar{q}_{2}}\right),
\end{aligned}
$$

where $p_{a \bar{b}}$ and $p_{c \bar{d}}$ are two of the mesonic quark-antiquark relative momenta, and $V_{\mathrm{D}_{i}}$ is the transition potential corresponding to diagram $\mathrm{D}_{i}$. The expression holds true for two-to-one, two-to-two, and two-to-three meson-meson scattering, but is assumed to be true for two-to-four meson-meson scattering, two-to-five meson-meson scattering, and so on. Let $m_{A}\left(m_{B}\right)$ be the mass of meson $A(B)$, and let $P_{j}$ and $J_{j}$ $\left(j=A, B, C_{1}, \cdots, C_{\mathrm{n}}\right)$ be the four-momentum and the angular momentum of meson $j$ with the magnetic projection quantum number $J_{j z}$, respectively. The unpolarized cross section for $A+B \rightarrow C_{1}+\cdots+C_{\mathrm{n}}$ is

$$
\begin{aligned}
\sigma^{\text {unpol }}(\sqrt{s}, T)= & \frac{1}{\left(2 J_{A}+1\right)\left(2 J_{B}+1\right)} \frac{(2 \pi)^{4}}{4 \sqrt{\left(P_{A} \cdot P_{B}\right)^{2}-m_{A}^{2} m_{B}^{2}}} \\
& \int \frac{d^{3} P_{C_{1}}}{(2 \pi)^{3} 2 E_{C_{1}}} \cdots \frac{d^{3} P_{C_{\mathrm{n}}}}{(2 \pi)^{3} 2 E_{C_{\mathrm{n}}}} \delta\left(E_{\mathrm{f}}-E_{\mathrm{i}}\right) \delta^{3}\left(\vec{P}_{\mathrm{f}}-\vec{P}_{\mathrm{i}}\right) \\
& \sum_{J_{A z} J_{B z} J_{C_{1} z} \cdots J_{C_{\mathrm{n}} z}}\left|\mathcal{M}_{\mathrm{D}_{1}}+\cdots+\mathcal{M}_{\mathrm{D}_{\mathrm{m}}}\right|^{2},
\end{aligned}
$$

\section{Summary}

We have seen that in solving physical problems precise parametrizations of scattering amplitudes of quite a few reactions have been given, and the $T$-matrix elements for inelastic meson-meson scattering are obtained from the Lippmann-Schwinger equations or by the generalization of the inverse amplitude method to multi channels. Starting from the $\chi \mathrm{PT}$ Lagrangians, the coupled-channel unitary approaches and the inverse amplitude method offer more insights into the strong interaction through meson-meson scattering. However, the approaches and the method based on meson degrees of freedom remain in vacuum.

For two mesons in the ground-state pseudoscalar octet and the ground-state vector nonet, their scattering may be caused at tree level by one or two of the processes: quark interchange, quark-antiquark annihilation, quark-antiquark creation, and quark-antiquark annihilation and creation. The transition potentials corresponding to quark-antiquark annihilation, quark-antiquark creation, and quarkantiquark annihilation and creation have been derived in QCD. From these processes two-to-one meson-meson scattering, two-to-two meson-meson scattering, and two-to-three meson-meson scattering have been studied. The transition amplitudes are composed of the transition potentials and the mesonic quark-antiquark wave functions of which the relative-motion part is the solution of the Schrödinger equation with the temperature-dependent quark potential. Unpolarized cross sections 
obtained from the transition amplitudes depend not only on the center-of-mass energy of the two initial mesons but also on the temperature of hadronic matter. From vacuum to hadronic matter the cross sections change considerably, and the influence of hadronic matter on inelastic meson-meson scattering is remarkable.

Finally, particular attention is paid to two current subjects revealed by experiments at the LHC. One subject is the large CP asymmetry in the charmless threebody decay modes of $B$ mesons. One needs to answer how large is the contribution of inelastic meson-meson scattering to the CP asymmetry, and whether future experiments can give more new decay modes in which the $\mathrm{CP}$ violation is influenced by inelastic meson-meson scattering. Another subject is the strong interaction between hadronic matter and a meson with a large momentum. For $\mathrm{Pb}-\mathrm{Pb}$ collisions at $\sqrt{s_{N N}}=5.02 \mathrm{TeV}$ the CMS datd ${ }^{49}$ and the ATLAS data $2^{50}$ show that the prompt- $J / \psi$ nuclear modification factor stays unchanged when the $J / \psi$ transverse momentum goes from $8 \mathrm{GeV} / c$ to $18 \mathrm{GeV} / c$. The unchanged nuclear modification factor is nontrivial! The mechanism where three or more mesons are produced in a collision of a light meson and a charmonium plays a key role in understanding the unchanged nuclear modification factor ${ }^{51}$ Inelastic meson-meson scattering is certainly important to a high-momentum meson penetrating hadronic matter.

\section{Acknowledgments}

This work was supported by the National Natural Science Foundation of China under Grant No. 11175111.

\section{References}

1. J. A. Oller and E. Oset, Nucl. Phys. A 620, 438 (1997).

2. I. V. Danilkin, L. I. R. Gil, and M. F. M. Lutz, Phys. Lett. B 703, 504 (2011).

3. Z.-H. Guo, L. Liu, U.-G. Meißner, J. A. Oller, and A. Rusetsky, Phys. Rev. D 95, 054004 (2017).

4. J. A. Oller and E. Oset, Nucl. Phys. A 629, 739 (1998).

5. I. Danilkin and M. Vanderhaeghen, Phys. Lett. B 789, 366 (2019).

6. I. Danilkin, O. Deineka, and M. Vanderhaeghen, Phys. Rev. D 101, 054008 (2020).

7. U.-G. Meißner and J. A. Oller, Nucl. Phys. A 679, 671 (2001).

8. F.-K. Guo, R.-G. Ping, P.-N. Shen, H.-C. Chiang, and B. S. Zou, Nucl. Phys. A 773, 78 (2006).

9. S. Sakai, W.-H. Liang, G. Toledo, and E. Oset, Phys. Rev. D 101, 014005 (2020).

10. LHCb Collaboration, Phys. Rev. D 90, 112004 (2014).

11. LHCb Collaboration, Phys. Rev. Lett. 123, 231802 (2019).

12. LHCb Collaboration, Phys. Rev. Lett. 124, 031801 (2020).

13. LHCb Collaboration, Phys. Rev. D 101, 012006 (2020).

14. J. H. A. Nogueira, I. Bediaga, A. B. R. Cavalcante, T. Frederico, and O. Luorenco, Phys. Rev. D 92, 054010 (2015).

15. P. Koch, B. Müller, and J. Rafelski, Phys. Rep. 142, 167 (1986).

16. W.-X. Li, X.-M. Xu, and H. J. Weber, Phys. Rev. D 101, 014025 (2020).

17. ATLAS Collaboration, Phys. Lett. B 790, 108 (2019).

18. CMS Collaboration, JHEP 10, 138 (2018). 
19. ALICE Collaboration, Phys. Lett. B 788, 166 (2019).

20. J. Gasser and H. Leutwyler, Ann. Phys. 158, 142 (1984).

21. J. Gasser and H. Leutwyler, Nucl. Phys. B 250, 465 (1985).

22. P. Herrera-Siklódy, J. I. Latorre, P. Pascual, J. Taron, Nucl. Phys. B 497, 345 (1997).

23. R. Kaiser and H. Leutwyler, Eur. Phys. J. C 17, 623 (2000).

24. M. Albaladejo, J. A. Oller, and L. Roca, Phys. Rev. D 82, 094019 (2010).

25. A. M. Torres, L. R. Dai, C. Koren, D. Jido, and E. Oset, Phys. Rev. D 85, 014027 (2012).

26. J. Weinstein and N. Isgur, Phys. Rev. D 41, 2236 (1990).

27. M. Albaladejo and J. A. Oller, Phys. Rev. Lett. 101, 252002 (2008).

28. J. A. Oller, E. Oset, and J. R. Peláez, Phys. Rev. Lett. 80, 3452 (1998).

29. J. A. Oller, E. Oset, and J. R. Peláez, Phys. Rev. D 59, 074001 (1999).

30. T. Barnes, arXiv:hep-ph/0202157.

31. T. Barnes, arXiv:hep-ph/0311102.

32. J. R. Peláez and A. Rodas, Eur. Phys. J. C 78, 897 (2018).

33. Y. S. Surovtsev, P. Bydžovský, T. Gutsche, R. Kamiński, V. E. Lyubovitskij, and M. Nagy, Phys. Rev. D 97, 014009 (2018).

34. J. J. Dudek, R. G. Edwards, and D. J. Wilson, Phys. Rev. D 93, 094506 (2016).

35. D. Lohse, J. W. Durso, K. Holinde, and J. Speth, Nucl. Phys. A 516, 513 (1990).

36. G. E. Brown, C. M. Ko, Z. G. Wu, and L. H. Xia, Phys. Rev. C 43, 1881 (1991).

37. A. M. Torres, K. P. Khemchandani, L. M. Abreu, F. S. Navarra, and M. Nielsen, Phys. Rev. D 97, 056001 (2018).

38. T. Barnes and E. S. Swanson, Phys. Rev. D 46, 131 (1992).

39. Y.-Q. Li and X.-M. Xu, Nucl. Phys. A 794, 210 (2007).

40. S.-T. Ji, Z.-Y. Shen, and X.-M. Xu, J. Phys. G 42, 095110 (2015).

41. W. Buchmüller and S.-H. H. Tye, Phys. Rev. D 24, 132 (1981).

42. F. Karsch, E. Laermann, and A. Peikert, Nucl. Phys. B 605, 579 (2001).

43. S. Godfrey and N. Isgur, Phys. Rev. D 32, 189 (1985).

44. X.-M. Xu, Nucl. Phys. A 697, 825 (2002).

45. Z.-Y. Shen and X.-M. Xu, J. Korean Phys. Soc. 66, 754 (2015).

46. Z.-Y. Shen, X.-M. Xu, and H. J. Weber, Phys. Rev. D 94, 034030 (2016).

47. T.-T. Wang and X.-M. Xu, Chin. Phys. C 43, 024102 (2019).

48. K. Yang, X.-M. Xu, and H. J. Weber, Phys. Rev. D 96, 114025 (2017).

49. CMS Collaboration, Eur. Phys. J. C 78, 509 (2018).

50. ATLAS Collaboration, Eur. Phys. J. C 78, 762 (2018).

51. S.-T. Ji, X.-M. Xu, and H. J. Weber, Nucl. Phys. A 966, 224 (2017). 